\title{
RDUS
}

Revue de DROIT UNIVERSITÉ DE SHERBROOKE

Titre : $\quad$ LA BONNE FOI : NOTION PROTÉIFORME

Auteur(s): $\quad$ Brigitte LEFEBVRE

Revue: $\quad$ RDUS, 1995-1996, volume 26, numéro 2

Pages: $\quad 321-354$

ISSN : $\quad 0317-9656$

Éditeur : $\quad$ Université de Sherbrooke. Faculté de droit.

URI : $\quad$ http://hdl.handle.net/11143/12870

DOI : https://doi.org/10.17118/11143/12870 
Page vide laissée intentionnellement. 


\section{LA BONNE FOI : NOTION PROTÉIFORME}

par Brigitte LEFEBVRE*

\section{SOMMAIRE}

SECTION 1: LA DUALITÉ DE LA NOTION DE BONNE FOI . . 323

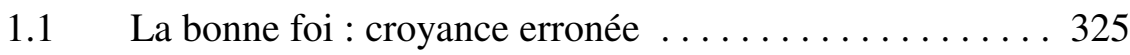

1.2 La bonne foi : norme de comportement ......... 328

1.2.1 L'élément psychologique . . . . . . . . . . . 329

1.2.2 L'élément moral . . . . . . . . . . . . . 333

1.2.2.1 La bonne foi et la responsabilité civile . . 336

1.2.2.2 La bonne foi et la faute civile ...... 338

SECTION 2: LES FONCTIONS DE LA BONNE FOI . . . . . . . 340

$2.1 \quad$ La fonction interprétative $\ldots \ldots \ldots \ldots \ldots \ldots \ldots \ldots \ldots$

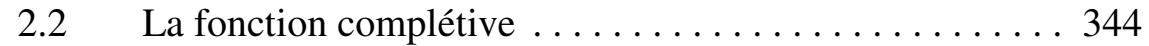

2.3 La fonction limitative ... . . . . . . . . . . . . . 346

2.4 La fonction adaptative .............. 352

*. $\quad$ Notaire et professeure au Département des sciences juridiques de l'UQAM. 
Étudier le contenu de la notion de bonne foi nous amène à nous demander : qu'est-ce que la bonne foi? La réponse à cette question est fort complexe car la notion de bonne foi n'est pas définie au Code civil. Cette absence de définition rend certes cette notion plus difficile à cerner, mais présente l'avantage de lui permettre d'évoluer et de s'appliquer à des situations nouvelles. ${ }^{1}$ C'est donc vers la doctrine et la jurisprudence que nous devons nous tourner pour faire l'étude de la bonne foi. Alors qu'il existe plusieurs études sur la bonne foi en droit français, ${ }^{2}$ le droit québécois est peu loquace sur le sujet. ${ }^{3}$

Dans un premier temps, nous nous pencherons sur la notion de bonne foi et nous constaterons qu'elle a plus d'un sens. Puis, dans un deuxième temps, nous examinerons l'utilité de la bonne foi en étudiant ses différentes fonctions.

\section{SECTION 1: LA DUALITÉ DE LA NOTION DE BONNE FOI}

La majorité des auteurs reconnaissent que la notion de bonne foi présente plusieurs facettes. ${ }^{4}$ La dualité de la notion de bonne foi s'entend généralement de la distinction faite entre la bonne foi - croyance erronée - et la bonne foi - norme de comportement. On utilise parfois le vocable «bonne foi

1. G. Cornu, Regards sur le titre III du livre III du code civil, «Des contrats ou des obligations conventionnelles en général» (essai de lecture d'un titre du Code), D.E.A. de droit privé, Paris, Les cours de droit, 1977 à la p. 59.

2. C. Arthaud, De la bonne foi et ses effets en matière civile, Paris, A. Parent, 1874; A. Breton, «Des effets civils de la bonne foi», [1926] Revue critique 86; R. Desgorces, La bonne foi dans le droit des contrats rôle actuel et perspectives, th. Paris II, 1992; F. Gorphe, Le principe de la bonne foi, Paris, Dalloz, 1928; J. Jaubert, Des effets civils de la bonne foi, Paris, A. Pédone, 1899; G. Lyon-Caen, «De l'évolution de la notion de bonne foi» (1946) 44 Rev. trim. dr. civ. 75; J. J. E. Millet, De l'erreur et de la bonne foi en droit romain et en droit français, Paris, Lahure, 1871; Y. Picod, Le devoir de loyauté dans l'exécution du contrat, t. 208, Paris, L.G.D.J., 1989; A. A.L. Volansky, Essai d'une définition expressive du droit basée sur l'idée de bonne foi, Paris, Librairie de jurisprudence ancienne et moderne, 1929; R. Vouin, La bonne foi : Notion et rôle actuels en droit privé français, Paris, L.G.D.J., 1939.

3. Congrès Henri Capitant - Journées Louisianaises 1992, (1992) 37 R. D. McGill 1024; S. Guillemard, «Tentative de description de l'obligation de bonne foi, en particulier dans le cadre des négociations précontractuelles» (1993) 24 R.G.D. 369; G. A. Rosenberg, «The notion of good faith in civil law of Quebec», (1960) 7 R. D. McGill 2.

4. $\quad$ Pour une revue de la doctrine française voir : R. Vouin, supra note 2 aux pp. 32-38; R. Desgorces, supra note 2 aux pp. 36-38. 
subjective» dans le premier cas et le vocable «bonne foi objective» dans le second. Le Vocabulaire Capitant fait ressortir cette dualité et définit la bonne foi en ces termes : «1) Croyance erronée en l'existence d'une situation juridique régulière. 2) Comportement loyal (ou à tout le moins normal) que requiert, notamment l'exécution d'une obligation; attitude d'intégrité et d'honnêteté.» ${ }^{5} \mathrm{En}$ matière contractuelle, un auteur a proposé une autre classification, qui distingue la bonne foi contractuelle de la bonne foi du contractant. ${ }^{6}$ La première se veut plus objective que la seconde, qui est intimement reliée à la personne du contractant. D'autres font une distinction entre une bonne foi dite active, qui consiste en l'intention de ne pas causer un dommage, et une bonne foi dite passive, qui consiste à être dans l'erreur. ${ }^{7}$ La distinction faite par Jaubert est toutefois essentiellement reliée à l'attitude psychologique de la personne. Comme nous le verrons, cette conception de la bonne foi n'est plus suffisante aujourd'hui. La notion de bonne foi n'est plus restreinte à l'étude de l'intention ou de la croyance d'un individu, mais implique également l'examen objectif de ses agissements en fonction d'un standard de comportement et ce, indépendamment de sa véritable intention.

Même si la notion courante de bonne foi est directement reliée à l'aspect psychologique d'un individu, la notion juridique de bonne foi quant à elle comprend généralement deux éléments : l'élément psychologique, intrinsèquement lié à l'individu et l'élément moral qui s'en détache, se rapportant plutôt aux valeurs de la société qu'à la morale individuelle. Ainsi, dans certains cas, l'aspect psychologique sera à lui seul déterminant, alors que dans d'autres cas, on tiendra compte de considérations extérieures, souvent à connotation morale, afin d'établir s'il y a bonne foi ou non. Parfois même, l'intention n'aura plus d'importance. Alors qu'à la base, la bonne foi est essentiellement une question d'ordre psychologique, nous constatons que, d'un point de vue juridique, cet aspect de la bonne foi s'estompe parfois pour laisser la place à des considérations extrinsèques.

Il faut donc faire une distinction entre être de bonne foi et agir selon les exigences de la bonne foi. Le fait de référer à la bonne foi dans les deux cas,

5. G. Cornu, dir., Vocabulaire Juridique, Association Henri Capitant, 5e éd., Paris, P.U.F., 1996 à la p.105; Voir aussi : P.-A. Crépeau, dir., Dictionnaire de droit privé et lexiques bilingues, $2^{\mathrm{e}}$ éd., Cowansville, Yvon Blais, 1991 à la p. 63.

6. Y. Picod, supra note 2.

7. J. Jaubert, supra note 2 à la p. 23. 
contribue à la complexité de la compréhension de cette notion, car cette dernière vise plus d'une réalité. Le vocable utilisé n'est pas approprié. Il aurait peut-être été plus judicieux de parler de bonne foi dans le premier cas, alors qu'il est question de l'intention, et de probité dans le second, lorsqu'on réfère à une norme de comportement.

On peut se demander si les deux acceptions de la notion de bonne foi existent en droit québécois. Nul doute ne saurait être soulevé à cet égard. Nous verrons toutefois que ces deux sens ne sont pas diamétralement opposés et exclusifs l'un de l'autre. Il existe certains éléments d'objectivité qui entrent en ligne de compte lorsque l'on considère la bonne foi sous son aspect subjectif, alors que certains éléments de subjectivité sont considérés lors de l'analyse de la bonne foi objective. Pour les fins de l'exposé, nous aborderons le sujet en utilisant la division faite entre la bonne foi - croyance-erronée, où l'élément psychologique prédomine, et la bonne foi - norme de comportement où les valeurs de la société, élément moral, joue un rôle important.

\subsection{La bonne foi : croyance erronée}

Être de bonne foi, c'est croire en l'existence d'une situation juridique régulière alors que la réalité est tout autre. Dans un tel cas, le droit porte secours à celui qui est de bonne foi, malgré l'irrégularité de sa situation réelle.

Cette notion traditionnelle de bonne foi - croyance erronée existe depuis fort longtemps en droit québécois. Une revue des écrits des principaux auteurs sous l'ancien code peut nous en convaincre. Pour Langelier, «la bonne foi c'est l'ignorance de quelque chose qu'on aurait besoin de connaître». ${ }^{8}$ Pour Rodys,

«la bonne foi c'est la juste opinion et croyance ferme qu'a le possesseur qu'il a acquis le domaine de propriété de la chose qu'il possède; c'est l'ignorance du droit d'autrui... c'est la croyance légitime... c'est une croyance pleine et entière de l'acquéreur dans le droit de propriété à lui transmis... c'est la conviction, découlant de l'ignorance de tout droit contraire». ${ }^{9}$

8. F. Langelier, Cours de droit civil de la province de Québec, t. 6, Montréal, Wilson et Lafleur, 1911.

9. W. Rodys, Traité de droit civil du Québec, t. 15, Montréal, Wilson et Lafleur, 1958; voir aussi : G. A. Rosenberg, supra note 3. 
Peu importe cependant que l'erreur soit de droit ou de fait. ${ }^{10}$ Force est de constater que cette bonne foi subjective a des liens étroits avec la notion d'erreur. Toutefois, on ne saurait les confondre. ${ }^{11}$ Jaubert fait remarquer que l'erreur et la bonne foi passive ont en commun de porter préjudice à celui qui se trompe. En effet, l'opinion fausse que l'on se fait entraîne des conséquences dommageables pour notre patrimoine. ${ }^{12}$ Cependant, le résultat obtenu lorsqu'on invoque l'erreur ou la bonne foi est différent. La bonne foi permet de maintenir une situation juridique irrégulière, alors que l'erreur sert à l'annuler. ${ }^{13}$ "Une personne de bonne foi est celle qui ignore que son adversaire est titulaire d'un droit ou qui croit être elle-même dans une situation juridique déterminée.» ${ }^{14} \mathrm{La}$ bonne foi dans une telle situation s'analyse par rapport à la partie qui tente de faire valoir ses droits et ne dépend aucunement des agissements de l'autre partie. On n'exige pas la démonstration que l'autre partie a été de mauvaise foi. Certains auteurs font remarquer que c'est l'élément croyance qui prédomine. ${ }^{15} \mathrm{C}$ 'est cette croyance fondée sur des prémisses erronées qui est la manifestation de la bonne foi. Pour d'autres, c'est l'erreur engendrée qui importe. ${ }^{16}$ Selon Vouin:

«La bonne foi est donc l'erreur sur l'irrégularité d'un acte ou d'une situation juridique, l'ignorance de cette irrégularité. Elle est une notion purement psychologique, et non morale. De là vient que soit sans influence la croyance à l'existence d'un vice qui, en réalité, n'existe pas.» ${ }^{17}$

Nous reconnaissons que l'erreur est l'élément prédominant dans cette notion subjective de bonne foi - croyance erronée, mais nous ne partageons pas l'opinion de l'auteur à l'effet que la bonne foi n'ait pas de connotation morale. Ce sont souvent la morale et les valeurs de la société qui vont servir à déterminer si l'erreur est constitutive de droit.

10. G. Lyon-Caen, supra note 2 à la p. 99; R. Vouin, supra note 2 à la p. 260; A. Breton, supra note 2 à la p. 91; C. Arthaud, supra note 2 à la p. 183.

11. R. Vouin, supra note 2 à la p. 240; J. Jaubert, supra note 2 à la p. 157.

12. J. Jaubert, supra note 2 à la p. 158.

13. J. Jaubert, supra note 2 à la p. 159; G. Lyon-Caen, supra note 2 à la p. 99.

14. G. Lyon-Caen, supra note 2 à la p. 99.

15. A. Breton, aupra note 2 à la p. 89; F. Gorphe, supra note 2 à la p. 118.

16. R. Vouin, supra note 2 à la p. 244; G. Lyon-Caen, supra note 2 à la p. 99.

17. R. Vouin, supra note 2 aux pp. 243-244. 
En effet, même si traditionnellement la notion de bonne foi tourne autour de l'idée de croyance erronée et qu'elle est intimement liée à l'état d'esprit du justiciable, elle ne peut s'analyser exclusivement en fonction de l'aspect psychologique. Malgré le silence du texte, déjà sous l'ancien code, on reconnaissait que pour avoir un effet juridique, l'erreur devait être plausible, raisonnable, excusable. ${ }^{18} \mathrm{Si}$ tel n'est pas le cas et ce, malgré sa bonne foi, le comportement de celui qui tente de s'en prévaloir est alors fautif. ${ }^{19}$ L'analyse de la bonne foi déborde alors du cadre personnel de l'individu qui l'invoque pour s'analyser plus objectivement en fonction des perceptions et des valeurs de la société et tend vers un standard social de comportement à respecter. Le magistrat amené à juger cette conduite porte alors un jugement de valeur qui n'a plus rien à voir avec la bonne foi de l'individu à proprement parler.

Cette idée du caractère raisonnable de l'erreur est reprise aujourd'hui dans le nouveau code en matière de vice du consentement, lorsqu'il exige pour mettre en oeuvre les dispositions de l'erreur que cette dernière soit excusable. ${ }^{20}$ Le juge analyse alors le comportement de la partie qui invoque l'erreur. Avec cette nouvelle règle en matière de vice du consentement, le droit québécois met l'accent sur les agissements d'une partie au lieu de faire primer la rencontre des volontés qui doit sous-tendre le rapport contractuel. Il s'agit d'une analyse plus objective, car elle n'exige pas une preuve d'intention. Elle est toutefois teintée d'une certaine subjectivité, car le juge fait rarement totalement abstraction de ses propres valeurs lorsqu'il a à décider ce qui constitue un comportement raisonnable ou non.

Il est intéressant de constater que l'excusabilité de l'erreur est souvent déterminée par rapport à l'obligation de renseignement qui pèse sur l'une des

18. L. Baudouin, Droit civil de la province de Québec, Montréal, Wilson et Lafleur, 1953 à la p. 427; P.-B. Mignault, Le droit civil canadien, t. 2, Montréal, C. Théoret, 1896 à la p. 484; A. Montpetit et G. Taillefer, Traité de droit civil du Québec, t. 3, Montréal, Wilson et Lafleur, 1945 à la p. 138; à noter qu'avant l'adoption du Code civil du Québec, l'erreur inexcusable pouvait toutefois donner ouverture à l'annulation du contrat sur la base d'erreur.

19. P.-B. Mignault, supra note 18 à la p. 484; Super Marché Lefort inc. c. Banque Nationale du Canada, [1987] R.L. 641 à la p. 649 (C.A.).

20. Le caractère excusable de l'erreur s'apprécie in concreto. J.-L. Baudouin, Les obligations, $4^{\mathrm{e}}$ éd., Cowansville, Yvon Blais, 1993, au no 167, à la p. 117; Paradis c. Merrett, $(20$ mars 1995), Québec 200-02-007957-931, J.E. 95-1039 (C.Q.). 
parties au contrat et par l'obligation de se renseigner qui pèse sur l'autre. ${ }^{21}$ Cette obligation a comme fondement la bonne foi. ${ }^{22}$

\subsection{La bonne foi : norme de comportement}

Être de bonne foi, c'est avoir un comportement honnête. Être de bonne foi, c'est également avoir un comportement loyal. Alors que l'honnêteté est intrinsèquement reliée à l'intention de son auteur, la loyauté peut s'entendre des agissements, des normes, des standards requis dans une situation donnée. «Est loyale, adéquate à la bonne foi, une situation juridique conforme aux "lois morales", fussent-elles seulement celles du juge». ${ }^{23}$ Sous cet angle, l'élément intentionnel s'estompe pour laisser place à un critère extrinsèque qui s'analyse par rapport aux standards de la société et non seulement en fonction de l'individu lui-même, ce qui a d'ailleurs été reconnu en jurisprudence. ${ }^{24}$ On parle souvent de bonne foi objective, car on examine le comportement d'un individu par rapport à une norme plutôt que par rapport à sa véritable intention. Toutefois, comme nous le verrons plus loin, l'élément psychologique continue de jouer un rôle important lorsque l'on traite de cette facette de la bonne foi.

21. Pour une illustration, voir : Paradis c. Merrett, ibid. et Maisons funéraires Blais inc. c. Ouellet, (3 avril 1995), Abitibi 615-02-000119-934, J.E. 95-1086 (C.Q.).

22. J. Alisse, L'obligation de renseignements dans les contrats, thèse de doctorat, Paris II, 1975 à la p. 60 [non publié]; F. Magnin, «Reflexions critiques sur une extension possible de la notion de dol dans la formation des actes juridiques, l'abus de situation», J.C.P. 1976.I.2780; Y. Boyer, L'obligation de renseignements dans la formation du contrat, Marseille, Presses universitaires Aix-Marseille, 1978 aux pp. 28 et 254; P. Jourdain, «Le devoir de "se" renseigner», D.1983.chron.139; C. Larroumet, Droit civil, t. 3, 2éd., Paris, Economica, 1990 à la p. 318; A. Seriaux, Droit des obligations, Paris, P.U.F., 1992 à la p. 74; J.-L. Baudouin, supra note 20 au no 276 à la p. 161; contra : P. Chauvel, Le vice de consentement, thèse de doctorat, Paris II, 1981 à la p. 226 [non publié]; La Cour suprême du Canada a reconnu l'application de la théorie de l'obligation de renseignement dans le droit québécois. L'honorable juge Gonthier fonde spécifiquement cette obligation sur le principe de la bonne foi. Banque de Montréal c. Bail ltée, [1992] 2 R.C.S. 554 à la p. 582; Voir aussi : F. \& I. Holdings Inc. c. 87313 Canada ltée, [1996] R.J.Q. 851 (C.A.).

23. P. Sanz De Alba, Sur quelques aspects de l'équité, thèse de doctorat, Université AixMarseille, 1980 à la p. 361 [non publié].

24. Vachon c. Lachance, [1994] R.J.Q. 2576 (C.S.); Godbout c. Entreprises J.G.F. Fiore inc., (25 octobre 1994), Montréal 500-05-005022-940, J.E. $94-1814$ (C.S.); Alexander-Maheu c. Banque Laurentienne du Canada, (21 décembre 1995) Montréal 500-05-004856-934, J.E. 96-547 (C.S.); Acier d'armature Rô inc. c. Stelco inc., [1996] R.R.A. 355 ( C.A.). 
La bonne foi - norme de comportement - est-elle une réalité en droit québécois? La lecture de certains articles du Code permet de répondre par l'affirmative. L'article 1375 C.c.Q. y fait clairement référence. Il déclare que : «La bonne foi doit gouverner la conduite des parties, tant au moment de la naissance de l'obligation qu'à celui de son exécution ou de son extinction.» À plus d'une reprise, le code relie expressément le caractère raisonnable d'un comportement ou d'une clause avec les exigences de la bonne foi. ${ }^{25}$ La question demeure toutefois de savoir ce qu'est «un comportement excessif et déraisonnable allant à l'encontre des exigences de la bonne foi?»

Nous étudierons donc cette notion de bonne foi objective en examinant dans un premier temps l'incidence de l'élément psychologique puis, dans un deuxième temps, son aspect extrinsèque qui est influencé par des considérations morales.

\subsubsection{L'élément psychologique}

L'élément psychologique joue un rôle important lorsqu'il s'agit de déterminer si une personne est de bonne foi ou non. En effet, dès que l'on réussit à démontrer que l'intention d'une personne est malhonnête, nous pouvons établir qu'elle n'est pas de bonne foi. Comme nous le verrons, l'inverse n'est pas vrai. Le problème pratique est de réussir à faire cette preuve d'intention qui s'avère parfois très difficile, voire quasi impossible. Pour pallier cette difficulté, le législateur a édicté une présomption de bonne foi. ${ }^{26}$ La bonne foi étant présumée, la mauvaise foi d'une personne devra être démontrée, ce que l'on fera en prouvant la connaissance. Cette preuve doit être prépondérante et s'établit en analysant l'ensemble du comportement de la personne visée. ${ }^{27}$

Ainsi, on traite la plupart du temps, plutôt de mauvaise foi que de la bonne foi. Ceci nous amène à nous demander si la bonne foi et la mauvaise foi ne sont que les deux facettes de la même notion ou plutôt si elles sont des réalités distinctes. ${ }^{28}$ Dans le langage courant, la bonne foi est l'antonyme de la mauvaise foi. Juridiquement, il semble que ce soit différent. En effet, si on se 
limite à l'analyse de l'état d'esprit d'un individu, une personne est soit de bonne foi ou de mauvaise foi. Toutefois, un individu peut agir à l'encontre des exigences de la bonne foi sans avoir d'intention malhonnête et tout en ayant un comportement moralement acceptable à ses yeux. L'élément psychologique estil indispensable à la preuve de la bonne foi? En d'autres termes, peut-on dissocier la notion de bonne foi de l'intention de nuire ou de tromper?

Avant de tenter de répondre à ces questions, quelques remarques s'imposent pour comprendre pourquoi l'étude de la bonne foi s'est faite dans le sillon de la mauvaise foi. La bonne foi a longtemps été analysée par rapport à son antonyme : la mauvaise foi. Au lieu de se demander si une personne était de bonne foi, on tentait plutôt de démontrer qu'elle était de mauvaise foi. Cette façon d'envisager le problème de la bonne foi est en partie reliée à l'existence de la présomption de bonne foi édictée par le code civil. Généralement, une partie n'a pas à démontrer sa bonne foi, car elle bénéficie de la présomption. C'est alors à celui qui prétend le contraire d'établir qu'elle n'est pas de bonne foi, c'est-à-dire qu'elle est de mauvaise foi.

On peut également expliquer cette situation par le fait que la bonne foi dans la formation des contrats s'est principalement développée autour de la notion de dol. Le dol véhicule une idée de tromperie et l'actio doli était à l'origine un recours de nature pénale, un délit qui résultait d'un comportement malhonnête. ${ }^{29}$

Pendant longtemps, le dol n'a pu résulter que de gestes positifs posés afin de tromper le cocontractant. Aujourd'hui, il sanctionne la mauvaise foi du cocontractant, que celle-ci provienne d'un geste positif, tromperie, mensonge, manoeuvre frauduleuse ou d'une omission, réticence ou silence. L'étude du dol nous amène inévitablement à examiner l'intention de tromper et, en conséquence, l'aspect psychologique d'un individu. Juridiquement, le dol est une erreur sciemment provoquée. Étant le résultat d'un agissement fautif, cette notion d'erreur n'est pas aussi restrictive que celle de l'article 1400 C.c.Q. Le droit n'a pas ici à protéger la stabilité des transactions, car l'annulation ou la réduction du contrat résulte des agissements du cocontractant ou est faite à sa connaissance et il ne saurait s'en plaindre. En conséquence, l'erreur vicie le

29. P. Bonassies, Le dol dans la conclusion des contrats, thèse de doctorat, Lille, 1955 [non publié]. 
consentement dans tous les cas. ${ }^{30}$ Que cette erreur soit provoquée par un geste positif tels une fausse représentation, un mensonge ou par un geste négatif, une omission ou un silence, l'intention de tromper, c'est-à-dire la faute intentionnelle, doit exister. Elle est de l'essence du dol. ${ }^{31}$ C'est l'élément intentionnel qui prime. ${ }^{32}$

L'utilisation du mécanisme juridique du dol devrait être limité aux cas où l'on peut établir cette intention de tromper et en conséquence, sanctionner la mauvaise foi. ${ }^{33}$ Nous partageons l'avis de M. Sériaux qui n'envisage pas que le dol puisse avoir une application en dehors du domaine de l'erreur provoquée. ${ }^{34}$ Il faut distinguer l'erreur de l'article 1400 de celle de l'article 1401. Hormis leurs champs d'application spécifiques, ce qui les distingue, c'est le fait que l'erreur soit volontairement provoquée dans le cas du dol. ${ }^{35}$ Il nous apparaît inconcevable que l'on puisse prétendre qu'une personne est de mauvaise foi sans intention en ce sens. Peut-être agit-elle incorrectement, déloyalement, à l'encontre des exigences de la bonne foi, mais n'en étant pas consciente, elle ne provoque pas sciemment une erreur et l'emploi de la notion de dol semble ici inapproprié. ${ }^{36}$

30. Art.1401 C.c.Q.

31. M. Tancelin, supra note 28 au no 129 à la p. 76; C. Garcin et O. Moreteau, «Le dol et l'obligation de renseignements dans la formation des contrats», [1982] Annales de la faculté de droit de Lyon 101 à la 112; J.-L. Baudouin, supra note 20 au no 174 à la p. 119; J. Ghestin, Traité de droit civil, t. 2, $2^{\mathrm{e}}$ éd., Paris, L.G.D.J., 1988 no 426 à la p. 457; J. Pineau, D. Burman et S. Gaudet, Théorie des obligations, $3^{e}$ éd., Montréal, Thémis, 1996, au no 86 à la p. 137.

32. P. Bonassies, supra note 29 à la p. 393.

33. La notion de dol est insuffisante pour couvrir toutes les situations où l'obligation de bonne foi est sanctionnée par le droit québécois. En effet, l'utilisation du dol devrait être réduite aux cas où l'erreur est provoquée intentionnellement. Toutefois, afin de couvrir certains cas de réticence non intentionnelle on a dénaturé ce recours. La notion de dol pourrait être élargie si on considérait le dol comme un vice du contrat. voir : P. Bonassies, supra note 29 aux pp. 409 et s.

34. Le dol ne devrait pas être étendu «à toutes les formes de malhonnêteté qui choquent la bonne foi contractuelle». A. Seriaux, supra note 22 à la p. 66.

35. L'obligation de bonne foi devrait quant à elle couvrir les cas ou une partie a un comportement déraisonnable sans qu'il soit toutefois intentionnel.

36. Le régime de la responsabilité civile serait plus opportun; toutefois il n'entraîne pas la même sanction car il ne permet pas d'obtenir l'annulation du contrat ou la réduction des obligations. Ceci explique probablement pourquoi le recours pour dol a, dans les faits, été le mécanisme juridique utilisé. 
Tous mensonges ou toutes réticences n'équivalent toutefois pas automatiquement à un dol. Il peut en effet s'agir d'un «bon dol», lequel est permis en droit. ${ }^{37}$ Le droit fait appel à un critère plus objectif qui réfère au comportement raisonnable et ne sanctionnera l'erreur provoquée que si elle est excusable. L'élément psychologique s'estompe encore une fois.

Quelle est la relation entre la bonne foi et la mauvaise foi et en quoi se distinguent-elles l'une de l'autre? La mauvaise foi est intimement liée à l'état d'esprit d'une partie, alors que la bonne foi ne requiert pas nécessairement une intention particulière. A priori, ceci peut sembler contradictoire. Volansky en donne une explication en examinant l'étymologie des mots bonne foi. «La notion de bonae fidei désigne à l'origine un usage : celui des honnêtes gens. Elle signifie donc une conduite générale qui se rapporte à un certain état d'esprit, mais ne vise pas seulement l'activité intentionnelle.» ${ }^{38}$ Certains ont proposé de résoudre cette contradiction en distinguant la bonne foi du contractant de la bonne foi contractuelle. ${ }^{39}$ Dans cette optique, la bonne foi du contractant serait une analyse in concreto de l'état d'esprit du contractant, ce qui revient à étudier la mauvaise foi, alors que la bonne foi contractuelle s'apprécierait in abstracto par rapport à une norme sociale reconnue.

Théoriquement, il apparaît possible d'isoler la notion de mauvaise foi de celle de la bonne foi - norme de comportement. M. le Juge Baudouin indique que la mauvaise foi doit être distinguée de la faute simple ou de la négligence en ce que «la mauvaise foi est plus qu'une simple faute ou négligence. Elle emporte, en principe, la connaissance effective de la réalité.» ${ }^{40}$ Dans une autre affaire, la Cour reconnaît qu'on ne perd pas sa bonne foi, c'est-à-dire qu'on est pas de mauvaise foi, parce qu'on a été imprudent. ${ }^{41}$ La mauvaise foi a donc indéniablement des liens très étroits avec l'état d'esprit d'une personne.

37. Lortie c. Bouchard, [1952] 1 R.C.S. 508; J.-L. Baudouin, supra note 20 au no 181 à la p. 122; J. Pineau, D. Burman et S. Gaudet, Théorie des obligation, supra note 31 au no 88 à la p. 139.

38. A. Volansky, supra note 2 à la p. 284.

39. R. Vouin, supra note 2 à la p. 145; Y. Picod, supra note 2 aux pp. 22 et 79.

40. Masella c. Nettoyeur Eden inc., [1993] R.J.Q. 1703 à la p. 1706 (C.A.).

41. Automobiles Mario Maratta inc. c. Meehan, (19 septembre 1994), Terrebonne 700-02002684-935, J.E. $94-1599$ (C.Q.). 
La bonne foi quant à elle, traite de la façon dont se comporte une personne. Comme nous le verrons dans la prochaine section, l'étude des fonctions de la bonne foi démontrera qu'entre autres, la bonne foi crée des obligations implicites pour les parties dans une relation contractuelle. Ainsi, lorsque le droit sanctionne des agissements en ne tenant pas compte de l'état d'esprit de son auteur mais plutôt en examinant la situation par rapports aux faits et gestes d'une personne raisonnable placée dans les mêmes circonstances, il sanctionne un manquement à l'obligation de bonne foi et non pas nécessairement la mauvaise foi d'une des parties.

L'expression mauvaise foi devrait être limitée aux cas où une intention malveillante existe. Force est de reconnaître que la jurisprudence ne limite pas l'utilisation du terme «mauvaise foi» aux situations où l'on rencontre cette intention caractérisée. À titre d'exemple, le juge Baudouin fait remarquer que quelquefois, lorsque la faute est qualifiée de lourde, la jurisprudence est portée à la faire équivaloir à de la mauvaise foi. ${ }^{42} \mathrm{Il}$ serait plus juste de dire qu'il y a faute lorsqu'on enfreint les exigences de la bonne foi. Ceci nous amènerait peut être à reconnaître que l'obligation de bonne foi a une existence autonome.

\subsubsection{L'élément moral}

Désormais, l'élément psychologique n'est plus le seul critère retenu afin d'établir s'il y a bonne foi ou non. La bonne foi se veut le critère qui détermine si un comportement est excessif et déraisonnable ou non. Elle est un standard de comportement. ${ }^{43}$ Un examen de la jurisprudence récente révèle cette nouvelle tendance. ${ }^{44}$ Dans l'affaire G.D.G. Environnement ltée c. Zoecon

42. Masella c. Nettoyeur Eden inc., supra note 40 à la p. 1706.

43. Cette évolution du droit existe également dans plusieurs pays civilistes. Travaux de l'Association Henri Capitant, La bonne foi, (Journées Louisianaises 1992), t. XLIII, Paris, Litec, 1994. Pour le droit canadien : E. P. Belobaba, «Good faith in canadian contract law», (1985) Spec. lect. L.S.U.C 73; S. K. O'Byrne, «Good faith in contractual performance : Recent developments», (1995) 74 R. du B. can. 70.

44. Posluns c. Entreprises Lormil inc., (4 juillet 1990), Québec 200-05-001584-858, 200-05001878-854, J.E. 90-1131 (C.S.) (en appel) Dans cette cause, on a reconnu que l'obligation de loyauté impliquait pour le locateur d'éviter les conflits d'intérêts entre les locataires. Dans les faits cette obligation avait pour effet de créer une obligation d'exclusivité implicite malgré l'absence de clause d'exclusivité au bail. Paradis c. Côté, (20 avril 1995), Chicoutimi 165-02-000193-943, J.E. 95-1163 (C.Q.). Dans cette affaire, le tribunal a examiné le comportement du débiteur afin de déterminer s'il avait agit de bonne foi ou non. Il n'a aucunement été question d'intention. 
Canada inc., le juge Croteau s'exprime en ces termes : «[...] la bonne foi est comprise dans le sens d'une norme, d'une conduite acceptable ou de l'exercice raisonnable d'un droit selon les règles de l'équité et de la loyauté.» ${ }^{45}$ Dans la même veine, la Cour suprême du Canada s'est prononcée sur le sujet dans une affaire où il était question d'abus de droit en matière contractuelle et a clairement établi qu'il peut y avoir abus sans qu'il soit nécessaire de prouver mauvaise foi ou malice. ${ }^{46}$ On examine alors le comportement en fonction de la personne raisonnable. En conséquence, agira à l'encontre des exigences de la bonne foi et abusera de ses droits, non seulement la personne qui a une intention malveillante ou malicieuse, mais également celle qui a eu un comportement déraisonnable dans les circonstances. Quelque temps plus tard, la Cour suprême s'est de nouveau penchée sur l'obligation de bonne foi dans une affaire portant sur la non-divulgation de données techniques dans le cadre de contrat d'entreprise à forfait. Dans cette affaire, la Cour a reconnu l'existence de l'obligation de renseignement et a statué qu'il y avait eu manquement à cette obligation en examinant le comportement du maître d'ouvrage à la lumière du comportement d'une personne raisonnable. ${ }^{47}$

Le Code civil du Québec a codifié l'interprétation donnée par la Cour suprême en matière d'abus de droit, à l'article 7. Nous remarquons donc que le code fait, dorénavant, expressément référence à la bonne foi lorsqu'il veut qualifier la façon d'exercer un droit.

Quoique l'élément psychologique demeure important lorsque l'on veut établir qu'une partie a agi avec une intention de nuire, force est de reconnaître cependant que l'intention du contractant s'estompe lorsqu'il s'agit de qualifier un comportement d'excessif ou de déraisonnable. Le juge se réfère alors à une norme abstraite conforme aux critères de ce qui est raisonnable aux yeux de la

45. (16 avril 1993), Montréal 500-05-002786-935, J.E. 93-1255 à la p. 9 (C.S.). Dans cette affaire, le tribunal est d'avis qu'une partie bénéficiant d'un droit d'exclusivité n'agit pas de bonne foi en faisant la promotion des produits d'un concurrent. On examine aucunement l'intention.

46. $\quad H o u l e$ c. Banque Canadienne Nationale, [1990] 3 R.C.S. 122.

47. Banque de Montréal c. Bail ltée, supra note 22. Notons toutefois que dans cette affaire le maître d'oeuvre avait connaissance des informations et qu'il ne les a pas transmises à son cocontractant. Il aurait alors été possible de faire la preuve de sa mauvaise foi. 
société et aux siens. ${ }^{48}$ C'est alors que des considérations morales entrent en ligne de compte. Le comportement du contractant sera analysé en fonction d'une personne raisonnable placée dans les mêmes circonstances. On pourra alors arriver à une situation où une personne sera réputée avoir agi à l'encontre des exigences de la bonne foi tout en n'ayant eu aucune intention en ce sens; ce sera notamment le cas de la personne totalement insouciante. Selon sa morale personnelle, son geste n'enfreint aucun code d'éthique alors qu'il est socialement répréhensible.

Il n'est donc plus nécessaire de se demander si la personne est selon ses propres convictions, de bonne foi ou non. On se demande plutôt si elle a agi selon les exigences de la bonne foi. Il y a donc une distinction fondamentale à faire entre être de bonne foi et agir selon la bonne foi. Cette distinction réside dans l'intention de l'individu. Si l'on est de mauvaise foi, on agit à l'encontre de la bonne foi, mais l'inverse n'est pas vrai. Il suffit maintenant de démontrer qu'une personne n'a pas agi de façon raisonnable, allant ainsi à l'encontre des exigences de la bonne foi. On doit toutefois en faire la preuve, vu la présomption de bonne foi édictée par l'article 2805. N'ayant plus à faire une preuve d'intention, la preuve en est facilitée et s'effectue par la démonstration de l'existence des faits en cause. Il suffit de prouver une faute simple. Mais est-il encore véritablement question de bonne foi dans son sens le plus courant du terme? N'est-il pas plutôt question de la personne raisonnable, ce qui nous amène à nous demander en quoi la bonne foi se distingue-t-elle de la responsabilité civile?

48. Dans l'affaire Vachon c. Lachance, supra note 24 à la p. 2579 (C.S.), M. le juge Allard s'exprime en ces termes : «Quelqu'un ne peut plus avoir de défense adéquate de bonne foi en prétendant seulement qu'il a agi légalement, qu'il est dans son droit ou qu'il se croyait dans son droit. Il devra, si sa bonne foi est contestée, aller plus loin et prouver qu'il a non seulement agi en toute légalité, mais conformément au standard social que la collectivité reconnaît.» 
1.2.2.1 La bonne foi et la responsabilité civile

Le principe de la bonne foi, à titre de norme de comportement, nous amène à nous interroger sur les liens qui peuvent exister entre cette notion et la responsabilité civile. Certains ont déjà soulevé l'existence de tels liens. ${ }^{49}$ En effet, l'une des idées maîtresses qui sous-tend la responsabilité civile est la violation d'une norme de conduite. ${ }^{50}$ Toutefois, la bonne foi ne doit pas être confondue avec la responsabilité civile. Nous devons voir la bonne foi comme étant le devoir à respecter et la responsabilité civile comme étant le mécanisme qui permet de sanctionner le manquement à ce devoir.

Entendu dans son sens subjectif, il semble y avoir peu de place pour les notions de bonne foi et de mauvaise foi dans le champs de la responsabilité extracontractuelle, car le droit québécois ne tient généralement pas compte de l'intention d'une partie afin de retenir sa responsabilité. Ceci rejoint les propos de Vouin qui ne voit pas de place pour la bonne foi dans la responsabilité délictuelle. $^{51}$ Nous devons nuancer cette affirmation, car la bonne ou la mauvaise foi d'un individu détermine la responsabilité dans certaines cas, notamment ceux visés par les articles 1461,1471 et 1474 C.c.Q. Elle peut également jouer un rôle lors de l'octroi de dommages punitifs (art.1621 C.c.Q.). En ce sens, la bonne foi est un facteur qui diminue l'obligation de réparation du préjudice subi. Cependant, il s'agit probablement plutôt d'un effet de la mauvaise foi que de la bonne foi dans son sens objectif.

Dans son sens objectif, il est, à priori, difficile de dissocier la bonne foi de la responsabilité civile. En tant que principe général de droit, la bonne foi trouve sa sanction dans la responsabilité civile. Un auteur propose que le principe de la bonne foi soit le fondement de la responsabilité civile. ${ }^{52}$ Pour sa part, le juge Gonthier de la Cour suprême considère que «l'obligation de bonne foi procède de la même source que l'obligation générale de bonne conduite

49. L. Cornelis, «La bonne foi : aménagement ou entorse à l'autonomie de la volonté», dans S. David-Constant, dir., La bonne foi, Liège, A.S.B.L., Jeune Barreau de Liège, 1990 à la p. 35; J. Ghestin, supra note 31 au no 185 à la p. 203.

50. J.-L. Baudouin, La responsabilité civile, $4^{\mathrm{e}}$ éd., Cowansville, Yvon Blais, 1994, au no 109 à la p. 82.

51. R. Vouin, supra note 2 aux pp. 197 et 201.

52. A. Volansky, supra note 2 à la p. 290. 
sanctionnée par l'article 1053 C.c.B.-C. (aujourd'hui art.1457 C.c.Q.).» ${ }^{53}$ Il parle d'ailleurs de «devoir général d'agir de bonne foi et de façon raisonnable». ${ }^{54} \mathrm{Il}$ existe donc inévitablement des liens étroits entre les deux notions. À ce titre, on peut s'interroger sur l'existence autonome du principe de la bonne foi. $\mathrm{Ne}$ fait-il pas double emploi avec la diligence requise par la responsabilité civile? Dans les deux cas, les tribunaux n'examinent-ils pas si l'individu a eu un comportement raisonnable? Afin de répondre à ces questions, il y a lieu d'examiner la question sous l'angle de la responsabilité contractuelle, puis sous l'angle de la responsabilité extracontractuelle. Cette question est importante, car s'il nous est impossible de distinguer la bonne foi de la responsabilité, elle n'apparaîtra pas comme une notion autonome et elle se confondra avec les règles de la responsabilité civile. Dans le cas contraire, la bonne foi apparaîtra comme une notion distincte ayant ses propres attributs et ses propres fonctions. Il nous semble donc primordial de déterminer s'il existe une obligation de bonne foi autonome, tant en matière contractuelle qu'extracontractuelle.

Le code civil québécois reconnaît spécifiquement à l'article 1375 , l'existence d'une obligation de bonne foi en matière contractuelle. Cette obligation existe-telle en dehors du cadre contractuel? Sans traiter spécifiquement de la question, certains écrits semblent abonder en ce sens. En effet, dans ses commentaires, le ministre de la Justice nous indique à l'article 1375 (qui vise l'obligation de bonne foi au stade de la formation, de l'exécution et de l'extinction de l'obligation) que cet article reprend, en matière d'obligations, le principe général édicté par l'article $6 .{ }^{55}$ A contrario, on peut interpréter les paroles du ministre comme reconnaissant que l'article 6 édicte un principe général de droit qui n'est pas limité au droit des contrats. Pour sa part, le professeur Masse évoque le fait que la notion de bonne foi en matières de responsabilité contractuelle et extracontractuelle doit être clarifiée. ${ }^{56} \mathrm{Au}$ surplus, nous pouvons argumenter que l'article 1375 fait partie du chapitre premier portant sur les dispositions générales du livre cinquième «Des obligations» et non dans le chapitre traitant des contrats. En conséquence, il devrait viser tant les obligations contractuelles que les obligations

53. Banque de Montréal c. Bail ltée, supra note 22 à la p. 582.

54. Ibid. à la p. 583.

55. Commentaires du ministre de la Justice, t. 1, Québec, Les publications du Québec à la p. 832.

56. C. Masse, «La responsabilité civile», dans La réforme du Code civil. Obligations, contrats nommés, vol. 2, Ste-Foy, P.U.L., 1993, au no 32 à la p. 260. 
extracontractuelles. Nous verrons ultérieurement que la bonne foi entretient des liens étroits avec la notion d'abus de droit. En effet, va à l'encontre de la bonne foi le fait d'exercer un droit de façon excessive et déraisonnable. Cette situation pouvant survenir tant en matière contractuelle qu'extracontractuelle, il ne saurait être contesté que la théorie de l'abus de droit trouve application dans les deux domaines. Il devrait en être de même du principe de la bonne foi.

Nous sommes d'avis que l'obligation de bonne foi devrait exister à l'extérieur du cadre contractuel. Comment peut-on exiger la bonne foi lors de la conclusion du contrat si on en nie l'existence durant la période où ce dernier se forme? Par ailleurs, ne pourrait-on pas voir dans l'article 6 qui exige que toute personne exerce ses droits civils selon les exigences de la bonne foi, l'existence d'une telle obligation légale? Nous le croyons. En effet, n'abuse-t-on pas de notre droit de contracter ou de ne pas contracter lorsque l'on rompt une négociation de façon intempestive? L'existence d'une obligation de bonne foi envers autrui a d'ailleurs été reconnue en jurisprudence. ${ }^{57}$

\subsubsection{La bonne foi et la faute civile}

La référence à la bonne foi comme standard de comportement nous amène à nous interroger sur la relation entre la bonne foi et la faute civile. De façon générale, les tribunaux ont recours à la notion de faute afin d'examiner les agissements des individus et d'établir s'il y a manquement aux exigences de la bonne foi. Le professeur Masse nous indique que la bonne foi se rapproche de la notion de faute. ${ }^{58}$ Toutefois, il ne faut pas confondre l'une et l'autre. À cet effet, rappelons les critères d'évaluation de la faute. Pour qu'il y ait faute, il faut premièrement qu'il y ait un manquement à un devoir imposé par la loi ou le contrat et, deuxièmement, que l'on apprécie le comportement à la lumière du critère de la personne raisonnable. La faute réside dans le comportement d'un

57. Métromédia C.M.R. inc. c. Tétreault, [1994] R.J.Q. 777 (C.S.). Dans cette affaire, le tribunal sanctionne les agissements d'un ex-employé et de son nouvel employeur et considère que la méconnaissance du préavis à donner lorsque l'on quitte un emploi est un comportement qui démontre une mauvaise foi si évidente qu'elle constitue un délit. Banque de Montréal c. Bail ltée, supra note 22 à la p. 582 : «[...] il va sans dire qu'une partie à un contrat doit se conduire tout aussi raisonnablement et avec la même bonne foi à l'égard des tiers qu'à l'égard des autres parties contractantes.»; D. Jutras, «Le tiers trompé (À propos de l'affaire Bail Ltée)», (1993) 72 R. du B. can. 28 aux pp. 40 et s.

58. C. Masse, supra note 56 au no 32 à la p. 261. 
individu, alors que la bonne foi est la norme en vertu de laquelle on juge ce comportement. Ainsi, sous certains aspects, la notion de bonne foi, notamment lorsqu'elle est envisagée comme critère d'application de l'abus de droit, devient un standard pour évaluer s'il y a faute. ${ }^{59}$

Pour sa part, M. Desgorces distingue nettement la bonne foi de la notion de faute. Cet auteur a démontré dans sa thèse que la bonne foi a une autonomie qui lui est propre, car il peut y avoir responsabilité contractuelle sans qu'il y ait faute. Selon lui, la bonne foi ne doit pas s'examiner comme une façon d'exécuter une obligation, mais plutôt comme étant l'objet même de l'obligation. ${ }^{60}$ Si cette interprétation peut être retenue en matière contractuelle, elle ne saurait l'être dans le domaine extracontractuel qui repose essentiellement sur le concept de faute.

Dans un autre ordre d'idées, si l'on examine la bonne foi dans son sens subjectif, c'est-à-dire en fonction de l'état d'esprit d'un individu, l'on doit avouer que la bonne foi ne peut être assimilable au concept de faute. Dans cette situation «la mauvaise foi est plus qu'une simple faute ou négligence, elle suppose la connaissance effective de la réalité.» ${ }^{61}$ La mauvaise foi est moindre et incluse dans la notion de faute. Il existe donc une certaine connexité entre la bonne foi et la faute civile sans qu'elles ne soient synonymes l'une de l'autre.

En conclusion, nous croyons qu'il est possible de distinguer l'obligation de bonne foi de la responsabilité civile en général. La bonne foi, comme critère d'évaluation de la faute, exigera parfois que l'on fasse plus que de s'abstenir de nuire à autrui. En effet, la bonne foi exige que l'on pose un geste positif. À titre d'exemples : informer la partie adverse, collaborer avec le cocontractant, éviter

59. Il a clairement été établi en commission parlementaire que l'intention du législateur est de ne donner ouverture au recours pour abus de droit que s'il y a faute et que cette théorie ne peut servir à introduire en droit québécois un régime de responsabilité sans faute. Québec, Assemblée nationale, Sous-commission des institutions, Journal des débats : étude détaillée du projet de loi 125 C.c.Q., 1ère session, 34e législature, Québec, Officiel, 1991 à la p. SCI1197.

60. R. Desgorces, supra note 2 à la p. 79; R. Vouin, supra note 2 à la p.43. Pour cet auteur, il est inadmissible de faire de la bonne foi une simple absence de faute. Contra : G. LyonCaen, supra note 2 à la p. 112 qui voit la bonne foi dans l'une des ses fonctions comme l'absence de faute contractuelle.

61. Masella c. Nettoyeur Eden inc., supra note 40; Automobiles Mario Maratta inc. c. Meehan, supra note 41. 
les conflits d'intérêts etc... En ce sens, la bonne foi crée des obligations positives, alors que la responsabilité extracontractuelle vise plutôt généralement à imposer une obligation de ne pas faire, de ne pas nuire à autrui. ${ }^{62} \mathrm{Vu}$ sous cet angle, la bonne foi apporterait un éclairage nouveau au cadre de la responsabilité civile délictuelle classique.

La jurisprudence nous permet de croire qu'il en est également ainsi en matière contractuelle. Mme la Juge Colas s'exprime en ces termes :

«Le rôle de la banque est de transiger de bonne foi afin de permettre à ses débiteurs de rencontrer leurs obligations en toute facilité et non de placer des obstacles pour paralyser l'exploitation et ainsi empêcher le redressement de la situation. Au lieu de jouer un rôle négatif, la banque se doit d'aider ses débiteurs à trouver des solutions susceptibles de redresser ou corriger certaines erreurs de parcours. ${ }^{63}$

La bonne foi implique donc un devoir de collaboration.

\section{SECTION 2: LES FONCTIONS DE LA BONNE FOI}

Comme nous venons de l'exposer, la bonne foi n'est pas une notion monolithique. Elle a plusieurs sens, ce qui laisse présupposer quelle n'intervient pas de la même façon et au même titre dans chacune de ses manifestations. Afin d'en comprendre les tenants et aboutissants, un examen de ses diverses fonctions s'avère opportun.

Pour les fins de l'exercice, nous avons choisi d'utiliser la classification proposée par le juriste belge Baert et reprise ici par le professeur Masse. ${ }^{64}$ Cette

62. J.-L. Baudouin, supra note 50 au no 30 à la p. 17.

63. Banque Royale du Canada c. Nettoyeur Terrebonne (1985) inc., (21 octobre 1987), Montréal 500-05-000006-872, J.E.88-61 (C.S.); Caisse populaire Desjardins St-JeanBaptiste-De-La-Salle c. 164375 Canada inc., [1996] R.R.A. 151 (C.S.) (en appel). Dans cette affaire, le tribunal a statué que le créancier devait notamment, pour se conformer à son obligation de bonne foi, fournir des explications plus détaillées et conseiller à son débiteur de consulter une tierce partie.

64. C. Masse, «La bonne foi dans l'exécution des contrats - Rapport Général», dans Travaux de l'Association Henri Capitant, La bonne foi, (Journées Louisianaises 1992), t. XLIII, Paris, Litec, 1994 aux pp. 224 et suiv. Cette classification n'est pas unanimement reconnue. Pour Matthias Storme, toutes ces distinctions sont inutiles car la bonne foi n'a qu'une fonction d'interprétation normative. Cité dans : L. Cornelis, supra note 49 à la p. 47. 
classification comprend quatre fonctions : une fonction interprétative, une fonction limitative, une fonction complétive et une fonction adaptative.

\subsection{La fonction interprétative}

La classification de Baert attribue à la bonne foi une fonction interprétative. Il semble que cette fonction occupe une place importante. ${ }^{65}$ En droit français tout comme en droit belge, elle trouve son fondement dans le troisième alinéa de l'article $1134 .{ }^{66} \mathrm{M}$. Périlleux expose que pour certains la fonction interprétative «commande que le contrat soit exécuté conformément à l'intention réelle des parties, l'esprit primant la lettre». ${ }^{67}$ Alors que pour d'autres, dont le professeur Masse, «cette première fonction permet au juge d'examiner la conduite des parties dans l'exécution du contrat pour voir si cette conduite est en accord avec les buts que les parties ne pouvaient manquer d'attribuer à ce contrat en vertu du principe de la bonne foi.» ${ }^{68}$ Il est alors plutôt question de comportement que d'intention.

Une première remarque s'impose. À la lecture de cette définition, il nous apparaît que l'on vise plus l'interprétation des comportements des parties au contrat que leur intention initiale. Cette fonction de la bonne foi ne permet donc pas de mieux connaître le contenu du contrat, mais plutôt d'analyser le comportement des contractants pour, le cas échéant, leur imposer des obligations non écrites. Ne parle-t-on pas plutôt de la bonne foi dans sa fonction supplétive qu'interprétative?

Quoiqu'il en soit, la fonction interprétative est-elle en accord avec les principes d'interprétation reconnus par le code civil? À première vue,

65. C. Masse, supra note 64 à la p. 224; voir la revue de la doctrine faite par Desgorces dans : R. Desgorces, supra note 2 à la p. 26. Certains ont soulevé des doutes sur le bien fondé de cette fonction. L. Cornelis, supra note 49 à la p. 49; R. Desgorces, supra note 2 aux pp. 31 et s.; J. Perilleux, «La bonne foi dans l'exécution du contrat. Rapport belge», dans Travaux de l'Association Henri Capitant La bonne foi, (Journées Louisianaises 1992), t. XLIII, Paris, Litec, 1994 à la p. 244.

66. L. Cornelis, supra note 49 à la p. 48.

67. J. Perilleux, supra note 65 à la p. 244.

68. C. Masse, supra note 64 à la p. 224. Pour les divers sens envisagés par la fonction interprétative de la bonne foi, voir : A. Benabent, «La bonne foi dans l'exécution du contrat. Rapport français», dans Travaux de l'Association Henri Capitant La bonne foi, (Journées Louisianaises 1992), t. XLIII, Paris, Litec, 1994 à la p. 294. 
permettons-nous d'en douter et ce, pour deux motifs. Premièrement, le Code édicte des règles aux articles 1425 et suivants qui servent à guider l'interprétation du contrat. On ne retrouve dans ces règles aucune trace de la bonne foi. Dans sa thèse, $\mathrm{M}$. Desgorces utilise entre autres cette constatation pour nier la fonction interprétative de la bonne foi. Il fait remarquer que l'article 1134 (articles 6, 1375 et 1434 en droit québécois), qui traite du contenu implicite d'un contrat, ne se retrouve pas dans la partie spécifiquement réservée aux règles d'interprétation des contrats. ${ }^{69}$ Mais est-ce suffisant pour exclure définitivement la bonne foi des principes d'interprétation des contrats? Nous y reviendrons.

Deuxièmement, les principes d'interprétation édictés au code civil posent comme première règle que le juge ne doit interpréter un contrat que si ce dernier est ambigu. Cette interprétation doit se faire en recherchant l'intention commune des parties et, en conséquence, faire prévaloir l'esprit du contrat sur la lettre..$^{70}$ À ce stade, ce qui importe, c'est de retracer ce qu'avait les parties en tête, ce sur quoi elles ont bien voulu s'entendre et non ce qu'elles ont censé avoir voulu en fonction d'éléments et de considérations externes. Il nous apparaît y avoir très peu de place pour la bonne foi dans cet exercice.

Le problème est donc de retracer cette commune intention, si commune intention il y a. En effet, les parties ont pu envisager leur contrat sous des angles bien différents l'une de l'autre. Lorsqu'il y a divergence d'opinion, le juge doit rechercher d'autres règles applicables afin de retracer «fictivement» la commune intention et c'est à ce moment là que peut être pris en considération le principe de la bonne foi. En effet, une certaine doctrine reconnaît que les règles d'interprétation ne se limitent pas exclusivement aux dispositions contenues dans la section visant spécifiquement l'interprétation des contrats. ${ }^{71}$ Il en existe

69. R. Desgorces, supra note 2 aux pp. 31 et 32.

70. Pour le professeur Malinvaud cette règle de base tire son inspiration de la notion de bonne foi. P. Malinvaud, Droit des obligations : les mécanismes juridiques des relations économiques, 6 éd., Paris, Litec, 1992 au no 170 à la p. 209.

71. J. Ghestin, supra note 31 au no 38 à la p. 42; E. Colas, «La notion d'équité dans l'interprétation des contrats», (1980-81) $83 R . d u$ N. 391 à la p. 400; Desgorces pour sa part, est en désaccord avec la fonction interprétative attribuée à l'article 1134 al. 3 . Il fait une nette distinction entre les problèmes d'interprétation et ceux de l'exécution, en considérant les problèmes d'interprétation comme étant ceux pour lesquels l'on doit déterminer ce que les parties ont voulu par opposition aux problèmes d'exécution qui visent le comportement des parties à un contrat. R. Desgorces, supra note 2 à la p. 31. 
d'autres. Il s'agit de règles dites objectives qui servent à déterminer le contenu des contrats. ${ }^{72}$ La bonne foi fait partie de ces règles objectives au même titre que l'usage et l'équité. ${ }^{73}$ Dans leur traité Colin et Capitant s'expriment ainsi :

«[...] s'il y a doute sur le sens de leur accord, celui-ci doit s'interpréter d'après les règles de la bonne foi, c'est-à-dire d'après le sens qui, en raison de la matière du contrat, s'imposerait normalement à un individu honnête, loyal en affaires.» ${ }^{74}$

Mais peut-on véritablement encore parler d'interprétation du contrat? ${ }^{75}$ C'est une chose que de déterminer le contenu d'une convention, c'en est une autre que de le rechercher exclusivement en fonction de l'intention des parties comme le propose le modèle classique. Lorsque l'intention est ambiguë ou que les intérêts des parties sont divergents, le résultat de l'analyse que fait le juge est faussé, car il doit reposer sur un élément, l'intention commune, qui est inexistant en l'espèce. Certes, l'introduction de ces principes objectifs se veut un palliatif à l'intention commune véritable, mais il nous apparaît qu'elle a plutôt pour effet de déterminer le contenu obligationnel du contrat sur une base autre que celle de l'intention commune des cocontractants. M. Cornelis abonde dans le même sens en les considérant comme des règles servant à «redéterminer l'intention commune des parties». ${ }^{76}$ Nous reconnaissons que le principe de la bonne foi est un facteur important lorsqu'il s'agit de déterminer le contenu d'un contrat, mais, selon nous, il ne saurait être vu et considéré comme une règle d'interprétation. Déterminer le contenu d'un contrat est une chose, l'interpréter en est une autre. Il y a là danger que, sous le couvert d'un mécanisme d'interprétation, le juge verse dans la révision du contrat. ${ }^{77}$ Dans les faits, il en est souvent ainsi; la limite entre l'interprétation du contrat et sa révision est vite franchie, ce qui nous amène plutôt à parler de fonction supplétive ou complétive de la bonne foi.

72. Voir en se sens : G. Leclerc, «La bonne foi dans l'exécution du contrat», dans Travaux de l'Association Henri Capitant La bonne foi, (Journées Louisianaises 1992), t. XLIII, Paris, Litec, 1994 à la p. 270; M. Tancelin, supra note 28 au no 227 à la p. 148.

73. Art. 1375 et 1434 C.c.Q.

74. A. Colin et H. Capitant, Traité de droit civil, t. 2, Paris, Dalloz, 1959 au no 814 à la p. 454.

75. J. Perilleux, supra note 65 à la 244.

76. L. Cornelis, supra note 49 à la p. 48.

77. Pour l'utilisation de la règle d'interprétation comme outil de révision du contrat, voir : P. Legrand jr, «L'obligation implicite contractuelle : aspects de la fabrication du contrat par le juge», (1991) 22 R.D.U.S. 109 aux pp. 133 et s. 


\subsection{La fonction complétive}

La fonction complétive de la bonne foi est reconnue et joue un rôle très important au niveau de l'exécution du contrat. ${ }^{78}$ «Cette notion permet de compléter les clauses et obligations assignées dans le contrat au débiteur par des devoirs et obligations accessoires qui vont l'obliger à exécuter utilement. ${ }^{79} \mathrm{En}$ ce sens, nous pouvons dire que la bonne foi est alors créatrice d'obligations. Certains ont pu prétendre qu'elle dénature le contrat et va à l'encontre du principe de l'autonomie de la volonté en imposant aux parties des obligations qu'elles n'ont pas expressément prévues; force nous est d'en reconnaître l'existence en droit québécois. ${ }^{80}$ Contrairement au droit français, nous avons vu précédemment que le Code civil du Bas-Canada ne contenait aucune référence expresse à la bonne foi dans l'exécution du contrat, ni au stade de la formation du contrat d'ailleurs. Cependant, l'article 1024 C.c.B.-C. stipulait que «les obligations d'un contrat s'étendent non seulement à ce qui y est exprimé, mais encore à toutes les conséquences qui en découlent, d'après sa nature, et suivant l'équité, l'usage ou la loi». Déjà, en 1965, un auteur proposait que la notion d'équité puisse donner ouverture à la reconnaissance de l'existence d'une obligation de bonne foi dans l'exécution des contrats. ${ }^{81}$ Cette idée a fait son chemin, tant en doctrine qu'en jurisprudence, et elle est maintenant expressément reconnue à l'article 1375 du Code civil du Québec. ${ }^{82}$ La fonction complétive de la bonne foi en matière d'exécution du contrat impose des obligations de collaboration, de loyauté, d'information. ${ }^{83}$

78. On utilise également le vocable «fonction supplétive» pour la désigner. L. Cornelis, supra note 49 à la p. 51 .

79. C. Masse, supra note 64 à la p. 225.

80. P. Legrand jr, supra note 77 aux pp. 109-154. Un auteur souligne que les obligations implicites reconnues en vertu de l'article 1024 C.c.B.-C. devraient se limiter aux obligations accessoires au type de contrat. D. Picotte, «Les mécanismes d'encadrement judiciaire des relations commerciales contractuelles et extra-contractuelles : évolution et tendances», (1993) 27 R.J.T. 599 à la p. 623.

81. P.-A. Crépeau, «Le contenu obligationnel d'un contrat», (1965) 43 R. du B. can. 1 à la p.26.

82. Banque de Montréal c. Bail ltée, supra note 22; Banque de Montréal c. Kuet Leong Ng, [1989] 2 R.C.S. 429 à la p. 436; Banque Nationale du Canada c. Soucisse, [1981] 2 R.C.S. 339 à la p. 357; J. Pineau et D. Burman, Théorie des obligations, $2^{\mathrm{e}}$ éd., Montréal, Thémis, 1988 au no 229 à la p. 316; J.-L. Baudouin, Les obligations, $2^{\mathrm{e}}$ éd., Cowansville, Yvon Blais, 1983 au no 369 à la p. 227.

83. C. Masse, supra note 64 à la p. 225; J. Perilleux, supra note 65 à la p. 244. 
En est-il de même au niveau de la formation du contrat? La bonne foi consiste notamment à assurer que le consentement va être donné de façon libre et éclairée. À cet effet, une obligation de renseignement peut être imposée à l'un des contractants en fonction de sa qualité, sur la base de la collaboration requise par la fonction complétive de la bonne foi. M. Boyer s'exprime ainsi : «La bonne foi implique cette forme de collaboration qui veut qu'un contractant renseigne l'autre contractant lorsqu'il existe entre eux un déséquilibre des connaissances.» ${ }^{84}$ Qu'en est-il en droit québécois? La Cour suprême, a rendu il y a quelques années un jugement fondamental sur le sujet. Après avoir retracé l'existence de plusieurs cas d'applications où l'on a exigé une obligation de renseignement, le cas du fabricant, le cas du médecin, en droit bancaire et en droit des assurances, le plus haut tribunal du pays a expressément reconnu l'existence en droit québécois d'une théorie générale de l'obligation de renseignement, ayant pour fondement la bonne foi dans le domaine contractuel. ${ }^{85}$ Cette obligation est positive, en ce sens que le débiteur de l'obligation se doit d'informer son cocontractant lorsque ce dernier est «dans une position informationnelle vulnérable. ${ }^{86}$ En obiter, la Cour reconnaît de plus que cette obligation ne se limite pas au domaine contractuel, mais qu'elle existe tout autant en matière extracontractuelle. ${ }^{87} \mathrm{Au}$ niveau jurisprudentiel, cette obligation de renseignement s'est notamment développée à travers la notion de réticence dolosive. ${ }^{88}$ Notons que le Code civil du Québec reconnaît expressément à son article 1401 que la réticence, voire même le silence, peuvent être constitutifs de dol. A contrario, il impose une obligation d'information. Comme nous l'avons souligné cependant, nous croyons que l'application de la bonne foi au stade de la formation du contrat ne saurait être limitée au concept de dol.

84. Y. Boyer, supra note 22 à la p. 20.

85. Banque de Montréal c. Bail ltée, supra note 22 à la p. 586.

86. Ibid. à la p. 587.

87. Ibid. à la p. 588.

88. À titre d'exemples récents : Landry-Caruso c. Marcotte, (4 novembre 1993), Montréal 50009-000875-898, 500-09-000876-896, J.E. 93-1878 (C.A.); Poissant c. Demers, (5 décembre 1994), Terrebonne 700-05-002306-912, J.E. 95-132 (C.S.); Marcotte c. Chastenay, (18 avril 1995), Trois-Rivières 400-05-000451-915, J.E. 95-1142 (C.S.), appel rejeté sur requête, 6 novembre 1995, C.A.Q. 200-09-000293-958; Waltzing c. Marchildon, (13 mars 1995), Chicoutimi 150-05-000469-918, J.E. 95-736 (C.S.), (en appel); 2435-5158 Canada inc. c. Canadian Kenworth Inc., (9 septembre 1994), Montréal 500-05-004213-888, J.E. 94-1459 (C.S.), règlement hors cour, $1^{\text {er }}$ juillet 1996, C.A.M. 500-09-001574-946; Nadeau c. Pelletier, (9 mai 1995), Longueuil 505-05-1452-934, J.E. 95-1350 (C.S.). 
La fonction complétive de la bonne foi impose un devoir de collaboration entre les parties contractantes. En l'occurrence, ne serait-il pas possible de prétendre que cette fonction de la bonne foi puisse donner ouverture à la reconnaissance d'une obligation de négocier de bonne foi? Notons que cette obligation existe dans le droit du travail. Elle figure dans la législation de façon expresse. ${ }^{89}$ L'obligation de négocier de bonne foi dans le droit du travail implique que les parties doivent négocier avec diligence dans le but d'en arriver à une entente, soit la conclusion d'une convention collective. ${ }^{90}$ L'obligation de négocier de bonne foi n'impose pas d'en arriver à une entente coûte que coûte. Si les négociations échouent, l'arbitre statuera ultérieurement sur les prétentions des parties. La situation est tout autre dans le droit commun des contrats. En effet, le principe de la liberté contractuelle n'oblige aucunement les parties qui entrent en pourparlers à conclure une entente et l'obligation de négocier de bonne foi peut apparaître comme allant fondamentalement à l'encontre de cette liberté contractuelle. Toutefois, l'existence de pourparlers, d'échange entre deux parties, nous semble suffisant pour justifier le fait de ne pas faire abstraction de cette phase. La formation d'un contrat ne débute pas avec la signature du contrat. Il ne saurait y avoir de naissance sans gestation. Il est donc primordial de ne pas ignorer la phase précontractuelle, qu'un contrat en résulte ou non. Si l'obligation de bonne foi peut exister en dehors de tout cadre contractuel, notamment en matière d'abus de droit, a fortiori devrait-elle exister lorsqu'une convention est susceptible de résulter de l'échange entre deux parties. La collaboration découlant de la fonction complétive de la bonne foi est susceptible d'y jouer un rôle.

\subsection{La fonction limitative}

La bonne foi a également une fonction limitative qui consiste à poser des limites à l'exercice d'un droit lorsqu'un tel exercice irait à l'encontre des exigences de la bonne foi et constituerait un abus de droit. M. Widmer parle pour sa part de la fonction modératrice de la bonne foi. À ses dires, cette fonction permet de «ramener l'exercice excessif d'un droit subjectif à un degré

\footnotetext{
89. Code du travail, L.R.Q., c. C-27 art. 53; Code canadien du travail, L.R.C. 1985, c. L-2 art. 50 a).

90. R. P. Gagnon, L. Lebel, P. Verge, Droit du Travail, 2e éd., Ste-Foy, P.U.L., 1991 à la p. 498.
} 
sûrement [...] qui peut être considéré comme légitime». ${ }^{91}$ Quant à M. Cornelis, il parle de fonction dérogatoire. ${ }^{92}$ Tous ces vocables visent la même réalité. Cette fonction de la bonne foi met clairement en évidence la relativité des droits.

Elle se retrouve codifiée à l'article 7 du code civil du Québec qui stipule : «Aucun droit ne peut être exercé en vue de nuire à autrui ou d'une manière excessive et déraisonnable allant ainsi à l'encontre des exigences de la bonne foi.» Cette phraséologie fait nettement ressortir les deux axes de l'abus de droit. Premièrement, l'article vise les situations où l'on est de mauvaise foi et qui sont directement reliées à l'intention de l'auteur. Deuxièmement, l'on traite plutôt des cas non intentionnels mais abusifs qui eux ne respecte pas le principe de la bonne foi. ${ }^{93}$ Nous voyons clairement que la bonne foi et la mauvaise foi ne sont pas toujours les revers d'une même médaille. La bonne foi agit ici comme «chien de garde», afin qu'un individu use de ses droits à bon escient. Cette théorie trouve des applications tant en matière contractuelle qu'extracontractuelle. $^{94}$

La bonne foi entretient des liens étroits avec la théorie de l'abus de droit. Toutefois, elles ne sauraient être assimilées l'une à l'autre. La bonne foi a un champ d'application beaucoup plus étendu que l'abus de droit qui n'en représente qu'un aspect. L'abus des droits peut se ramener à une manifestation du principe général de la bonne foi. ${ }^{95}$

Les liens entre l'abus de droit et la bonne foi sont manifestes dans le domaine contractuel. À l'origine, la théorie de l'abus de droit n'était pas admise en matière contractuelle, car on ne pouvait concevoir qu'un droit expressément

91. P. Widmer, «Bonne foi et abus de droit - Principe? Protée? Panacée? Une tentative de synthèse possible.» Séminaire sur «l'abus de droit et le principe de la bonne foi», Olivone, Suisse, septembre 1992 à la p. 8.

92. L. Cornelis, supra note 49 à la p. 52.

93. L'examen de la jurisprudence nous démontre toutefois que les tribunaux rendent leurs jugements en fonction du caractère déraisonnable ou non du comportement et font peu référence à la bonne foi.

94. Le code en rétière le principe à l'article 976 en matière de relation de bon voisinnage. La doctrine est toutefois d'avis, que cet article se distingue de la notion d'abus de droit codifiée à l'article 7 et qu'il consacre le critère de l'utilisation antisocial d'un droit, ce qui a pour effet d'introduire un mécanisme de responsabilité sans faute. J.-L. Baudouin, supra note 50 au no 161 à la p. 108.

95. M. Tancelin, Des obligations : contrat et responsabilité, $4^{\mathrm{e}}$ éd., Montréal, Wilson et Lafleur, 1988 au no 446 à la p. 267. 
prévu au contrat puisse être susceptible d'abus. ${ }^{96}$ La doctrine, principalement Josserand, a contribué à mettre en évidence que les droits ne sont pas absolus, mais plutôt relatifs. ${ }^{97}$ En effet, il survient fréquemment des conflits entre divers droits qui ont comme conséquence d'entraîner des limitations aux dits droits et d'en démonter leur relativité. ${ }^{98} \mathrm{Il}$ en est de même pour les conflits entre les règles contractuelles et les exigences de la bonne foi, ce qui occasionne des limitations, des modifications ou des interdictions. Dans un tel cas, M. Cornelis est d'avis que les règles de la bonne foi doivent prévaloir sur les règles contractuelles. ${ }^{99}$ Cette conclusion se justifie amplement selon nous, car le principe de la bonne foi est hiérarchiquement supérieur.

Peu à peu, tant la doctrine que la jurisprudence, ont reconnu que l'on pouvait abuser d'un droit contractuel. ${ }^{100}$ L'application de la théorie de l'abus de droit en matière contractuelle ne fait plus aucun doute depuis l'arrêt Houle c. Banque Canadienne Nationale rendu par la Cour suprême du Canada en 1990. Après avoir fait une revue exhaustive du droit français et québécois sur le sujet, Madame le Juge L'Heureux-Dubé conclut en ces termes : «En résumé, donc, il semble que la théorie de l'abus des droits contractuels fasse aujourd'hui incontestablement partie du droit québécois.» ${ }^{101}$ Cet arrêt met en évidence le lien qui existe entre l'abus de droit contractuel et le principe de la bonne foi.

96. Planiol était un ardent défenseur de cette thèse. Voir : J. Ghestin et G. Goubeaux, Traité de droit civil : Introduction générale, $3^{\mathrm{e}}$ éd., Paris, L.G.D.J., 1990 au no 695 à la p. 676.

97. L. Josserand, De l'esprit des droits et leur relativité, théorie dite de l'abus des droits, $2^{\mathrm{e}}$ éd., Paris, Dalloz, 1939.

98. L. Baudouin, supra note 18 à la p. 1278.

99. L. Cornelis, supra note 49 à la p. 53.

100. D. Angus, «Abuse of rights in contractual matters in the province of Quebec» (1962) $8 R$. D. Mc Gill 150; A. Mayrand, «L'abus des droits en France et au Québec» (1974) 9 R.J.T. 321 aux pp. 331 et s.; R. Soucy, «Étude sur les abus de droits», [1979] R.L. 1; J.-F. Desbiens, «L'abus de droit en matière contractuelle au Québec», (1987) 1 R.J.E.L. 81; P.-G. Jobin, «Grands pas et faux pas de l'abus de droit contractuel», (1991) 32 C. de D. 153; C. Vennat, «Commentaire d'arrêt : Banque Nationale du Canada c. Houle», (1988) 22 R.J.T. 387; G. Vallée et E. Naufal-Martinez, «La théorie de l'abus de droit dans le domaine du travail», dans G. Trudeau, G. Vallée et D. Veilleux, dir., Études en droit du travail : à la mémoire de Claude D'Aoust, Cowansville, Yvon Blais, 1995 à la p. 303.

101. Houle c. Banque Canadienne Nationale, supra note 46 à la p. 164. Cet arrêt a été commenté dans les articles suivants : C. Masse, «Chronique de droit civil québécois : session 1989-90» (1991) 2 (2d) Supreme Court L. R. 493; D. Gardner, «Un jalon dans l'établissement d'un nouvel ordre contractuel? : Houle c. Banque canadienne nationale», (1991) 70 R. du B. can. 760; Rosalie Jukier, «Banque Nationale du Canada v. Houle (S.C.C.) : Implications of an Expanded doctrine of abuse of rights in civilian contract law», (1992) 37 R. D. Mc Gill 221. 
C'est, en effet, par l'intermédiaire du principe de la bonne foi que l'abus de droit contractuel a été introduit en droit québécois. ${ }^{102}$ Le principe de bonne foi impose que les contrats soient exécutés de bonne foi et qu'en conséquence, on n'abuse pas de nos droits contractuels. La bonne foi a ainsi pour fonction de contrôler les droits contractuels.

Il devient donc opportun d'établir les conditions d'application de la notion de l'abus de droit en droit québécois. Encore une fois, la bonne foi s'avère un élément important. Il est désormais établi que le droit québécois ne retient plus uniquement l'obligation de démontrer l'existence d'une faute intentionnelle pour appliquer la notion de l'abus de droit contractuel. ${ }^{103}$ Dans l'affaire Houle c. Banque Canadienne Nationale, le plus haut tribunal du pays a non seulement consacré la théorie de l'abus de droit en matière contractuelle, mais a également établi qu'il y abus de droit, non seulement lorsqu'on exerce un droit avec une intention de nuire ou de manière malicieuse, mais également lorsqu'on exerce un droit contractuel de façon déraisonnable. Madame le juge L'Heureux-Dubé conclut en ces termes :

«[...] la norme servant à apprécier l'existence d'un tel abus s'est élargie pour inclure maintenant le critère de l'exercice raisonnable d'un droit, tel qu'il est incarné dans la conduite d'une personne prudente et diligente. Ce critère peut couvrir un grand nombre de situations, y compris l'utilisation d'un contrat à une fin autre que celle envisagée par les parties. On pourrait donc formuler ainsi le critère approprié : tels droits ont-ils été exercés dans un esprit de loyauté?» ${ }^{104}$

Le critère de l'abus de droit réfère non seulement à la bonne foi-intention mais également à la notion de bonne foi objective. La bonne foi devient alors une norme de comportement et l'intention s'estompe. C'est d'ailleurs ce critère qui a été repris par le législateur québécois lors de la codification de la théorie de l'abus de droit dans le code civil.

102. J.-L. Baudouin, «Contrat - application de la théorie de l'abus de droit en matières contractuelles», (1971) 31 R. du B. 335; C. Masse, supra note 64 à la p. 225.

103. Pour l'exposé des différentes thèses sur le sujet, voir: L. Mazeaud, «La responsabilité dans l'exercice d'un droit», (1955-56) 58 R. du N. 369 aux pp. 370-371; J. Ghestin et G. Goubeaux, supra note 96 aux pp. 691 et s.

104. Houle c. Banque Canadienne Nationale, supra note 46 à la p. 164. 
Dans ce passage, la Cour suprême souligne au surplus que l'abus de droit vise également les cas où un droit est utilisé pour une fin autre que celle envisagée par les parties. Dans une affaire subséquente, le juge Gendreau de la Cour d'appel réitère le test énoncé par la Cour suprême. Il s'exprime ainsi :

«C'est pourquoi la partie qui prétend à l'abus de droit devra démontrer que les gestes de son cocontractant sont inspirés par des considérations extérieures ou non pertinentes, ou dénotent une conduite déraisonnable eu égard à celle de l'individu prudent et diligent placé dans des conditions semblables à celles de la partie recherchée en dommages.» ${ }^{105}$

La référence à «des considérations extérieures ou non pertinentes» ne revient-elle pas à dire que l'on tient également compte de la finalité des droits et du caractère légitime ou non du motif? Le discours tenu par la jurisprudence pourrait, nous semble-t-il, donner ouverture, en partie du moins, à la théorie de Josserand. ${ }^{106}$ Cependant, les discussions qui ont eu lieu préalablement à l'adoption de l'article 7 du code civil, démontrent que la codification de la théorie de l'abus de droit correspond aux enseignements de la jurisprudence et qu'elle n'a pas pour effet d'introduire un mécanisme de responsabilité sans faute. ${ }^{107}$ Cette opinion est partagée par la doctrine ${ }^{108}$ et généralement suivi par la jurisprudence qui, dans la plupart des cas, se prononce expressément quant à l'exigence d'établir une faute ou qui, à tout le moins, le fait implicitement en

105. Sofati ltée c. Laporte, [1992] R.J.Q. 321 à la p. 325 (C.A.) Ce critère a été repris afin de déterminer s'il y avait abus de droit dans l'affaire Duchesne c. Produits Canadiana inc., $\left(1^{\mathrm{er}}\right.$ novembre 1994), Montréal 500-09-001458-900, J. E. 94-1904 (C.A.).

106. Selon Josserand, «la théorie de l'abus présente en réalité une double face, l'une subjective, l'autre objective, puisqu'elle implique la confrontation des motifs de l'agent - élément personnel et subjectif - avec la fonction, le but du droit en cause - élément social et objectif.» L. Josserand, supra note 97 au no 266 à la p. 366. Pour l'auteur, la notion de «motif légitime» n'est pas synonyme de mobile et ne requiert pas une intention blâmable. Ibid. à la p. 413.

107. Journal des débats, supra note 59 aux pp. SCI-1197 et 1198. Le Barreau du Québec avait souligné l'importance de retenir le critère de la faute en matière d'abus de droit. Barreau du Québec, Mémoire du Barreau du Québec sur le C.c.Q. (P.L. 125) Livre 1 : Des personnes, août 1991.

108. J.-L. Baudouin, supra note 50 au no 161 à la p. 108; C. Masse, supra note 56 au no 31 à la p. 259. 
référant à un comportement raisonnable. ${ }^{109}$ Les propos de M. le juge Gendreau de la Cour d'appel en font foi : «Cela dit, je ne crois pas, sauf preuve d'une conduite fautive, que la théorie de l'abus de droit autorise un contractant à faire mettre de côté ou à demander de rendre inopérante une disposition contractuelle à laquelle il a validement consenti.» ${ }^{110}$

Toutefois, cette prise en considération par la Cour d'appel du but du contrat nous permet d'entrevoir un élargissement du critère du caractère déraisonnable d'un droit tout en demeurant dans le cadre de la faute. Il nous apparaît que l'examen qui est fait porte avant tout sur le comportement, mais que l'on tiendra compte pour évaluer le caractère abusif de ce comportement d'un critère objectif, en l'occurrence le but de contrat. C'est dans cette optique que le critère de la finalité du droit a été retenu récemment. ${ }^{111}$ Dans cette affaire, le ministère du Revenu, afin de recouvrir une créance contre une compagnie insolvable, a usé de menaces pour d'obtenir par une voie détournée un cautionnement de la part d'une des actionnaires de la compagnie. Le tribunal constate que, même si les moyens de pression exercés pouvaient être légitimes, le but, lui, ne l'était pas, car l'actionnaire n'avait aucunement l'obligation de se rendre personnellement responsable des dettes de la compagnie. Dans cette affaire toutefois, même si l'on a considéré un critère objectif, la preuve démontre que l'on a également tenu compte du comportement du demandeur qui, aux dires du tribunal, était empreint de mauvaise foi et de malice. Il ne s'agit donc pas d'un cas de responsabilité sans faute.

109. À titre d'exemples, notamment : Banque Nationale du Canada c. Morneau, [1994] R.R.A. 944 (C.S.); Standard Broadcasting Corp. c. Stewart, [1994] R.J.Q. 1751 C.A.); Banque de Nouvelle-Ecosse c. Exarhos, [1995] R.R.A. 4 (C.A.); Vachon c. Lachance, [1994] R.J.Q. 2576 à la p. 2579; Équipements E. Lamontagne ltée c. Équipements Belarus du Canada ltée, (3 octobre 1994), Roberval 155-05-000189-937, J.E. 94-1684 (C.S.); Banque Royale du Canada c. Papaspyrou, J.E. 94-791 (C.S.). Contra : le juge Chouinard, s'exprimant au nom de la Cour d'appel est d'avis que l'abus de droit contractuel est maintentant reconnu même en l'absence de faute. E. et S. Salsberg Inc. c. Dylex Ltd., [1992] R.J.Q. 2445 (C.A.) Dans une autre affaire, on a retenu comme critère celui du comportement inéquitable. Martin c. Trizec Equities Ltée, (18 juin 1992), Montréal 500-05-006724-866, J.E. 92-1262 (C.S.). Ces décisions sont restées isolées.

110. Sofati ltée c. Laporte, supra note 105 à la p. 325 (C.A.).

111. Québec (sous-ministre du revenu) c. Caron, [1992] R.J.Q. 1084 (C.S.). 


\subsection{La fonction adaptative}

La fonction adaptative de la bonne foi s'illustre principalement à travers la théorie de l'imprévision. ${ }^{112}$ Elle permet de modifier un contrat et de le rééquilibrer lorsque des circonstances imprévues viennent en cours de route modifier les règles du jeu et qu'il ne correspond plus à l'intention initiale des parties. Un auteur a su démontrer que le principe de la bonne foi donnait ouverture à la réception de la théorie de l'imprévision et qu'il en était le fondement. ${ }^{113}$

«La bonne foi veille à ce que le contrat réponde aux attentes réciproques des parties contractantes; elle pèse les intérêts en présence, elle tient compte de la situation particulière de chacun et requiert la collaboration dans la réalisation des objectifs que les parties se sont fixés.» ${ }^{114}$

Au niveau de la formation du contrat, la fonction adaptative de la bonne foi intervient lorsqu'il est question de clauses abusives. Le Code civil, à l'article 1437, réprime dans les contrats d'adhésion et les contrats de consommation, les clauses abusives. Il permet alors au juge de réviser à la baisse ou d'annuler une clause qui est excessive et déraisonnable et qui va à l'encontre de la bonne foi. Il est alors question de la fonction limitative de la bonne foi. La bonne foi limite la liberté contractuelle en modifiant en partie un contrat et en ne donnant pas effet à toutes ses clauses.

A priori, on pourrait prétendre que le caractère excessif et déraisonnable doit être examiné uniquement en tenant compte de la situation des parties lors de la conclusion du contrat et considérer les clauses abusives comme étant une

112. De façon générale, cette doctrine n'est pas admise en droit québécois. Le principe général de la bonne foi pourrait toutefois permettre de la reconnaître. J.-L. Baudouin, supra note 20 aux $\mathrm{n}^{\text {os }} 424$ et 425 aux pp. 244-245. Voir également pour l'utilité de la notion de bonne foi dans la reconnaissance de la théorie de l'imprévision : S. Martin, «Pour une réception de la théorie de l'imprévision en droit positif québécois», (1993) 34 C. de D. 599 à la p. 624.

113. D. M. Philippe, Changements de circonstances et boulversements de l'économie contractuelle, Bruxelles, Bruylant, 1986. Voir également: Y. Picod, supra note 2 aux pp. 212 et $\mathrm{s}$.

114. D. M. Philippe, «Rapport belge - La bonne foi dans la formation des contrats», dans Travaux de l'Association Henri Capitant, La bonne foi, (Journées Louisianaises 1992), t. XLIII, Paris, Litec, 1994 à la p. 64. 
illustration de la fonction limitative attribuée à la bonne foi. On ne voit alors aucun lien entre les clauses abusives et la fonction adaptative de la bonne foi. Toutefois, l'examen de la jurisprudence révèle cependant une toute autre réalité. Afin d'établir si une clause est déraisonnable ou non, les tribunaux ne tiennent pas uniquement compte de la situation des parties lors de la conclusion du contrat, mais tiennent également compte des conséquences de ladite clause sur la situation du cocontractant (au moment où l'action est intentée). ${ }^{115}$ Ainsi, dans certains cas, ils adaptent le contrat en fonction d'une situation qui n'existait pas lors de la conclusion du contrat. Le caractère excessif ou déraisonnable dépend donc souvent de faits postérieurs à la formation du contrat, tout comme c'est le cas en matière d'imprévision.

On peut s'interroger sur la légalité d'une telle démarche. Selon nous, le caractère abusif d'une clause à un contrat est une question concernant la formation du contrat et devrait s'analyser par rapport à cette époque. En effet, si le législateur permet au juge d'intervenir en matière de clause abusive, c'est parce que l'une des parties au contrat n'a pas eu l'opportunité de faire valoir ses droits lors de la négociation du contrat et qu'elle a dû accepter cette clause. $\mathrm{Vu}$ le déséquilibre des forces en présence, la partie forte a su dicter sa loi. La jurisprudence, en tenant compte d'éléments postérieurs à la conclusion du contrat, traite les clauses abusives comme étant une question d'exécution du contrat. Ceci a pour conséquence d'occasionner une révision du contrat et de donner un certain effet à la théorie de l'imprévision qui, à ce jour, n'est pas reconnue formellement en droit québécois.

Ainsi, malgré le fait que l'on puisse s'interroger sur la légitimité de ce processus, force est de constater que les agissements de la jurisprudence reconnaissent en matière de formation des contrats une fonction adaptative à la bonne foi.

En conclusion, cet exercice a révélé que la bonne foi n'est pas une notion monolithique. On la conçoit principalement dans notre droit soit comme une croyance erronée, soit comme une norme de comportement et on lui attribue

115. À titre d'illustrations : 151276 Canada inc. c. Verville, [1994] R.J.Q. 2950 (C.S.); Société générale Beaver inc. c. Métaux ouvrés St-Philippe inc., (19 juillet 1994), Montréal 500-05016209-924, J.E. 94-1295 (C.S.), (en appel); Janin Construction (1983) ltée c. Régie d'assainissement des eaux du bassin de Laprairie, (23 septembre 1994), Montréal 500-05008375-907, J.E. 94-1559 (C.S.), (en appel). 
plusieurs fonctions. On a de plus constaté que la bonne foi ne s'analyse pas uniquement en fonction de son élément psychologique ou intentionnel. Elle tend à rejoindre le standard d'évaluation du comportement de la responsabilité civile qui est celui de la personne raisonnable, ce qui introduit dans son appréciation des considérations d'ordre moral. C'est une chose que d'être de bonne foi et c'en est une autre que d'agir conformément à la bonne foi. La bonne foi se dissocie donc de l'intention pour revêtir un aspect plus objectif. 